\title{
STRESS ANALYSIS OF ECCENTRIC RINGS IN BIPOLAR COORDINATE SYSTEM
}

\author{
Visaj Kandarpkumar Pandya ${ }^{1}$, Payal Desai ${ }^{2}$ \\ ${ }^{1}$ Graduate Student, School of Science and Engineering, Navrachana University, Bhaily Vasna Road Vadodara, 391 \\ 410, Gujarat \\ ${ }^{2}$ Assistant Professor, School of Science and Engineering, Navrachana University, Bhaily Vasna Road Vadodara, 391 \\ 410, Gujarat
}

\begin{abstract}
Stress analysis of eccentric ring is carried out in bipolar coordinate system under uniform internal and external pressure. Airy stress function is chosen in such a way that it satisfies the bi-harmonic equations and boundary conditions in bipolar coordinate system. Stresses are obtained for various parameters such as eccentricity, various inner radius to outer radius ratios. Effect of eccentricity is investigated on the stresses of the eccentric rings. Results are useful for the design of eccentric rings.
\end{abstract}

Keywords: Eccentric Rings, Bipolar Coordinate Systems, Computational Mechanics, Airy stress function, Structural Mechanics

\section{INTRODUCTION}

Rings or cylinders with eccentric hole are widely used in engineering structure. These holes are usually cause the change of stresses, displacements and decrease the load carrying capacity. The two dimensional problem of stress distribution in an elastic isotropic disk containing an eccentric circular hole has been studied in this paper by using Airy stress function in bipolar coordinates system. Mathematical formulation for elastic stress fields in bipolar coordinate systems are seen in (Desai and Kant (2015)). Aim of the present study is to investigate the effect of the hole on the stresses in eccentric rings at the vicinity of the hole.

\section{PROBLEM FORMULATION DESCRIPTION}

AND

The problem of eccentric rings subjected to uniform internal and external pressure is considered and shown in Fig. 1. and defined as bi-polar coordinates. We assume rings are made up of elastic material. The elastic ring has inner radius $r$, outer radius $\mathrm{R}$ and eccentricity $\mathrm{e}$, also both member are subjected to the uniform pressure. The inner and outer contours of the eccentric ring are defined by $\beta_{1}$ and $\beta_{2}$, respectively.
$* * *$

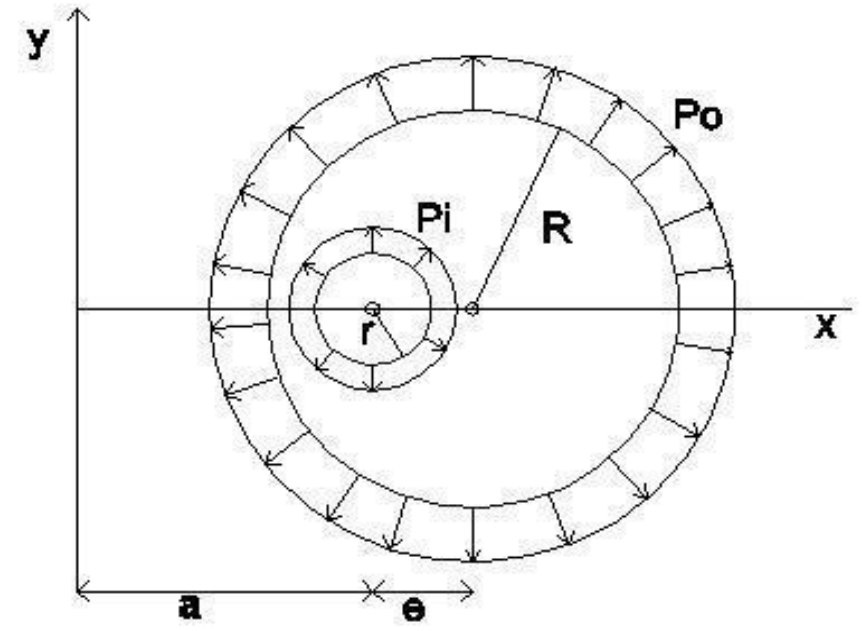

Fig. 1. Bipolar coordinates systems for problem of a disk/ring of radius $\mathrm{R}$ containing an eccentric circular hole of radius $r$ in a disk.

$$
\begin{gathered}
\beta_{1}=\cos ^{-1} h \frac{\left[\left(R^{2}\right)-\left(r^{2}\right)-\left(e^{2}\right)\right]}{2 e r}, \\
\beta_{2}=\cos ^{-1} h \frac{\left[\left(R^{2}\right)-\left(r^{2}\right)+\left(e^{2}\right)\right]}{2 e R}
\end{gathered}
$$

The length a and the thickness of the ligament $\lambda$ are given by

$$
a=\frac{1}{2 e} \sqrt{\left(R^{2}+r^{2}-e^{2}\right)}-4 r^{2} R^{2},
$$




$$
\lambda=R-r-e, \quad \frac{e}{R}=0.6
$$

Where, $a$ is a positive length and $\mathrm{e}$ is an centre to centre distance between outer circle and inner circle. Inner(r) and outer $\operatorname{radius}(\mathrm{R})$ of the circle can be defined bya $/ \sinh \beta$

\section{STRESS IN BIPOLAR COORDINATES}

The problem is formulated using an Airy stress function , which satisfied the biharmonic equation (Radi et. Al. (2009), Chen (2011)),

$$
\frac{\delta^{4} h \mathrm{x}}{\delta \beta^{4}}+2 \frac{\delta^{4} \mathrm{hx}}{\delta \alpha^{2} \delta \beta^{2}}+\frac{\delta^{4} \mathrm{hx}}{\delta \alpha^{4}}+2 \frac{\delta^{2} \mathrm{hx}}{\delta \beta^{2}}-2 \frac{\delta^{2} \mathrm{hx}}{\delta \alpha^{2}}+\mathrm{hx}=0
$$

Where,

$$
\begin{aligned}
& h x=\varphi / a^{2}(\cosh \alpha-\cos \beta) \\
& \varphi=A \log \beta+B \beta^{2} \log \beta+C \beta^{2}+D
\end{aligned}
$$

It is modified stress function. Corresponding stress are given by the relations:

$$
\sigma \beta=\frac{\partial^{2} h x}{\partial \beta^{2}}(\cosh \alpha-\cos \beta)-\frac{\delta h x}{\delta \alpha} \sinh \alpha-\frac{\partial h x}{\partial \beta}(\sin \beta)+h x \cosh \alpha
$$

$$
\sigma \alpha=\frac{\partial^{2} h x}{\partial \alpha^{2}}(\cosh \alpha-\cos \beta)-\frac{\delta h x}{\delta \alpha} \sinh \alpha-\frac{\partial h x}{\partial \beta}(\sin \beta)+h x \cos \beta
$$

$$
\tau \beta \alpha=-\frac{\delta^{2} h x}{\delta \beta \delta \alpha}(\cosh \alpha-\cos \beta)
$$

The Stress distribution is symmetric with respect to the $\mathrm{X}$ axis, the modified stress function hx can be derived from most general equation of the bi-harmonic equation in bipolar coordinates, which contains only derivative terms in $\beta$, so Shear stress $\tau_{\beta \alpha}$ become Zero, in the present case because of uniform pressure. Airy stress function becomes (Timoshenko (1970)),

$$
\varphi=A \log \beta^{2}+C \beta^{2}
$$

$\mathrm{P}_{\mathrm{i}}$ and $\mathrm{P}_{0}$ the uniform internal and external pressures. Then boundary conditions are (Timoshenko (1970)),

$$
\begin{array}{r}
(\sigma)_{\beta 1}=-P_{i} \\
(\sigma)_{\beta 2}=-P_{\mathrm{o}}
\end{array}
$$

Unknown constants $\mathrm{A}$ and $\mathrm{C}$ are found from the boundary conditions and substituted into the equations (5) and (6)

$$
\begin{aligned}
& \sigma \beta=A / a^{2}\left[\left(-\frac{\cosh \alpha^{2}}{\beta^{2}}\right)+(\log \beta \cos \beta \cosh \alpha)+\left(2 \sin \beta \frac{\cosh \alpha}{\beta}\right)+\left(\cos \beta \frac{\cosh \alpha}{\beta^{2}}\right)+\right. \\
& \left(\cosh \frac{\cos \beta}{\beta^{2}}\right)-(\log \beta)\left(\cos \beta^{2}\right)-(2 \sin \beta \cos \beta / \beta)-\left(\cos \beta^{2} / \beta^{2}\right)-(\cosh \alpha)(\sin \beta) / \\
& \left.\beta-(\log \beta)\left(\sin \beta^{2}\right)+(\cos \beta \sin \beta / \beta)+\log \beta\left(\cosh \alpha^{2}\right)-(\log \beta \cos \beta \cosh \alpha)\right]+ \\
& C / a^{2}\left[2\left(\cosh \alpha^{2}\right)-(2 \cos \beta \cosh \alpha)+(4 \beta \sin \beta \cosh \alpha)+\left(\beta^{2} \cos \beta \cosh \alpha-\right.\right. \\
& (2 \cosh \alpha \cos \beta)+2\left(\cos \beta^{2}\right)-(4 \beta \sin \beta \cos \beta)-\left(\beta^{2}\right)\left(\cos \beta^{2}\right)- \\
& (2 \beta)(\cosh \alpha \sin \beta)-(2 \beta \cos \beta \sin \beta)+\left(\beta^{2}\right)\left(\sin \beta^{2}\right)+\left(\beta^{2}\right)\left(\cosh \alpha^{2}\right)- \\
& \left.\left(\beta^{2}\right) \cos \beta \cosh \alpha\right] \\
& \sigma \alpha=\frac{A}{a^{2}}\left[(\log \beta)\left(\cosh \alpha^{2}\right)-(\log \beta)(\cosh \alpha)(\cos \beta)-\left(\frac{\cosh \alpha}{\beta}\right) \sin \beta-(\log \beta)\left(\sin \beta^{2}\right)+\right. \\
& \left.\frac{\cos \beta \sin \beta}{\beta}+(\log \beta \cosh \alpha \cos \beta)-(\log \beta)\left(\cos \beta^{2}\right)\right]+\frac{c}{a^{2}}\left[\left(\beta^{2}\right)\left(\cosh \alpha^{2}\right)-\left(\beta^{2}\right)(\cos \beta)-\right. \\
& \left.(2 \beta)(\cosh \alpha) \sin \beta+(2 \beta)(\cos \beta)(\sin \beta)-\left(\beta^{2}\right)(\cosh \alpha \cos \beta)+\left(\beta^{2}\right)\left(\cos \beta^{2}\right)\right]
\end{aligned}
$$




$$
\tau_{\beta \alpha}=0
$$

Stresses can be found out using equation (10) and (11) with the help of constant value $\mathrm{A}$ and $\mathrm{C}$.

\section{RESULTS AND DISCUSSIONS}

Numerical value and variation of stresses $\sigma_{\alpha}$ and $\sigma_{\beta}$ are shown in Fig.2. For the case when $\mathrm{a}=5 \mathrm{~m}, \beta=0.246 \mathrm{~m}$ for inner circle and $\beta=1 \mathrm{~m}$ for outer circle. Result are obtained for $\mathrm{R}=8 \mathrm{~m}, \mathrm{r}=2 \mathrm{~m}, \beta_{1}=1.27 \mathrm{~m}, \beta_{2}=0.40 \mathrm{~m}$ and $\sigma=\pi / 3$ radian. Value of inner and outer pressure is 1 psi or 6894.75 $\mathrm{N} / \mathrm{m}^{2}$. It is found from Fig. 2 that value of $\sigma_{\alpha}$ and $\sigma_{\beta}$ increases with $\beta$ (coordinate which is a logarithmic ratio between the two focal length of two circles, Arfken (2011)) near the boundaries of the eccentric rings. When, $\beta=\beta_{2}$, Eq. (10) gives $\sigma_{\beta}=\mathrm{P}_{\mathrm{o}}$

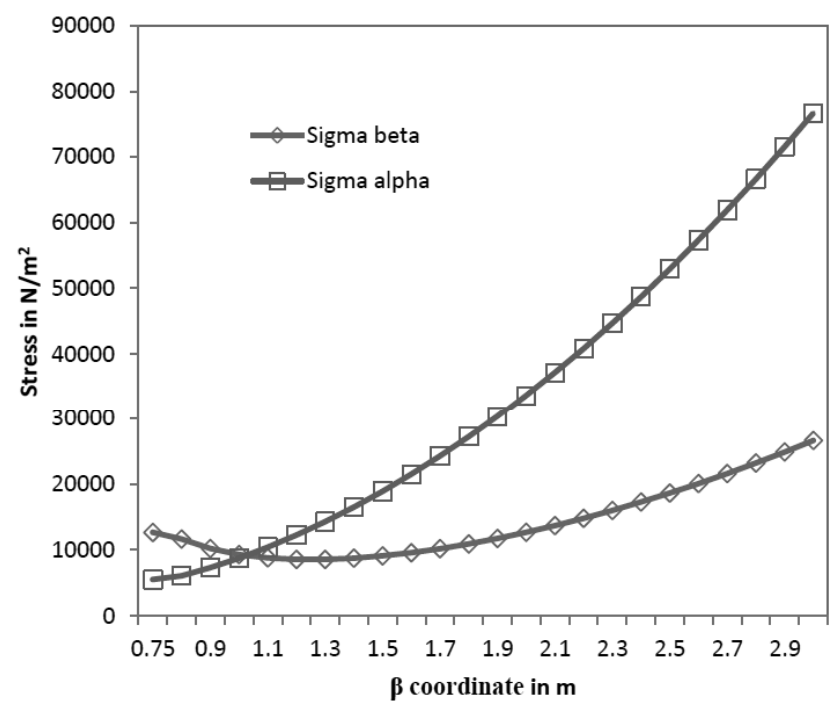

Fig. 2 Variation of stresses $\sigma_{\alpha}$ and $\sigma_{\beta}$ through the thickness and the bipolar coordinate $\beta$

\section{CONCLUSION}

The analytical solution for stresses has been carried out in an eccentric ring with circular hole subjected to uniform internal and external pressure. Airy's stress function is used which satisfies the biharmonic equation in bipolar coordinate system. Stresses are obtained in bipolar coordinate system. The results are new and can be used for designing eccentric rings subjected to uniform internal and external pressure.

\section{REFERENCES}

[1] E. Radi, A. Strozzi., "Jeffery solution for an elastic disk containing a sliding eccentric circular inclusion assembled by interference fit." International Journal of Solid and Structures 46(2009) 4515-4526

[2] J.T Chen et al., "Engineering Analysis with boundary Elements 35(2011) 236-243

[3] P. Desai, T. Kant., "On Derivation of Stress Field in Bi-polar Coordinates Systems", Advances in Structural Engineering, Springer India, India, 2015
[4] GB Arfken "Mathematical methods for physicists", 6th edition, Academic Press, San Diego, 2011

[5] SP Timoshenko, JN Goodier, "Theory of Elasticity" McGraw Hill Education inc. 3rd edition, 1970. 